\title{
Manifestações Cutâneas Associadas à COVID-19: Uma Revisão Narrativa
}

\section{Cutaneous Manifestations Associated with COVID-19: A Narrative Review}

\author{
Maria RELVAS $\triangle^{1}$, Joana CALVÃO ${ }^{1}$, Raquel OLIVEIRA ${ }^{2}$, José Carlos CARDOSO ${ }^{1}$, Margarida GONÇALO ${ }^{1,3}$ \\ Acta Med Port 2021 Feb;34(2):128-136 - https://doi.org/10.20344/amp.14574
}

\section{RESUMO}

Introdução: A COVID-19, doença infeciosa causada por um novo coronavírus, SARS-CoV-2, propagou-se rapidamente pelo mundo inteiro, resultando numa necessidade emergente de obtenção de conhecimentos alusivos à sua patogénese, diagnóstico e sintomatologia. Mais especificamente, um número cada vez maior de casos relativos a manifestações cutâneas, previamente desconhecidas, tem vindo a ser descrito.

Material e Métodos: Foi realizada uma pesquisa de literatura, através da base de dados PubMed, referente às manifestações dermatológicas associadas à COVID-19. O presente artigo descreve os achados clínicos e histológicos das principais lesões cutâneas observadas em contexto da infeção por SARS-CoV-2.

Discussão: Manifestações cutâneas associadas à COVID-19 foram descritas em múltiplos estudos retrospetivos e prospetivos, séries de casos e casos clínicos isolados. A incidência reportada atingiu os $20,4 \%$, verificando-se uma heterogeneidade de padrões clínicos substancial. Destes destacam-se as erupções eritematosas/maculopapulares, urticariformes, papulovesiculares, purpúricas/petequiais, lesões tipo-perniose e lesões livedóides/acro-isquémicas. O conhecimento dos mecanismos fisiopatológicos subjacentes tem vindo a ser enriquecido com achados histológicos e de biologia molecular.

Conclusão: É essencial o reconhecimento das manifestações dermatológicas associadas à COVID-19, uma vez que podem permitir o diagnóstico precoce da infeção, nomeadamente em casos oligossintomáticos ou quando não é possível a realização de testes confirmatórios. Embora menos estabelecido, lesões tipo-perniose e acro-isquémicas, parecem ter também um papel importante a nível prognóstico.

Palavras-chave: COVID-19; Infecções por Coronavírus; Manifestações Cutâneas; SARS-CoV-2

\section{ABSTRACT}

Introduction: COVID-19 is an infectious disease caused by the new coronavirus, SARS-CoV-2, that has spread rapidly throughout the world. This has resulted in an urgent need to obtain information regarding its pathogenesis, diagnosis and clinical manifestations. More specifically, skin manifestations, seldom reported initially, have been increasingly described.

Material and Methods: We performed a literature search in the PubMed database, regarding cutaneous manifestations associated with COVID-19. This article describes the clinical and histological findings of the main skin lesions observed in the context of SARS-CoV-2 infection.

Discussion: Cutaneous manifestations associated with COVID-19 have been described in multiple retrospective and prospective studies, case series and case reports. The reported incidence reached $20.4 \%$. Although there was substantial heterogeneity in terms of clinical patterns, the main ones include: erythematous/maculopapular, urticarial, papulovesicular, and purpuric/petechial eruptions, chilblain-like lesions and livedoid/acro-ischemic lesions. In the vast majority, the underlying pathophysiologic mechanisms are not fully understood, although histopathological findings and biomolecular studies can add relevant data.

Conclusion: The recognition of cutaneous manifestations associated with COVID-19 is of utmost importance. They may help establishing an early diagnosis, namely in oligosymptomatic patients or when confirmatory tests are impossible to perform. Moreover, chilblain-like lesions and acro-ischemia, also seem to play an important role in terms of prognosis.

Keywords: Coronavirus Infections; COVID-19; SARS-CoV-2; Skin Manifestations

\section{INTRODUÇÃO}

Em dezembro de 2019, na cidade chinesa de Wuhan, surgiram relatos dos primeiros casos de uma doença infeciosa do sistema respiratório provocada por um novo coronavírus, denominado 'severe acute respiratory syndrome coronavirus 2' (SARS-CoV-2). Pouco tempo depois, foi anunciado oficialmente pela Organização Mundial de Saúde (OMS) o novo nome desta patologia, que viria a propagar-se pelo mundo inteiro: Coronavirus disease 2019 (COVID-19). ${ }^{1}$

Em março de 2020 a Europa tornou-se o epicentro da pandemia de COVID-19, declarada pela OMS no dia 11 desse mês. Desde então, quase 60 milhões de casos foram confirmados resultando num total de mais de 1 milhão de mortes relacionadas com a doença. ${ }^{2}$ Portugal conta com quase 300 mil casos e mais de 4000 mortes. $^{3}$

O SARS-CoV-2 é maioritariamente transmitido por via respiratória, através de gotículas contaminadas, embora as vias fecal-oral, sexual ou vertical possam também constituir possíveis formas de contágio. ${ }^{4} \mathrm{O}$ tempo de incubação médio é de cinco dias sendo que $97,5 \%$ dos infetados

1. Serviço de Dermatologia e Venereologia. Centro Hospitalar e Universitário de Coimbra. Coimbra. Portugal.

2. Serviço de Medicina Interna. Centro Hospitalar e Universitário de Coimbra. Coimbra. Portugal.

3. Departamento de Dermatologia. Faculdade de Medicina. Universidade de Coimbra. Coimbra. Portugal.

$\triangle$ Autor correspondente: Maria Relvas.mariavrelvas@gmail.com

Recebido: 15 de julho de 2020 - Aceite: 26 de novembro de 2020 - First published: 14 de dezembro de 2020 - Online issue published: 01 de fevereiro de 202 Copyright $\odot$ Ordem dos Médicos 2021 
irá apresentar sintomas nos primeiros $12 .{ }^{5}$ A confirmação diagnóstica da COVID-19 é fornecida através da deteção de RNA viral por real time polymerase chain reaction ou pela presença de anticorpos IgM e/ou IgG específicos para SARS-CoV-2, em indivíduos com história epidemiológica e/ ou manifestações clínicas sugestivas de infeção. ${ }^{6}$

Embora habitualmente associada a quadros de infeção das vias respiratórias superiores e pneumonia, a COVID-19 apresenta-se clinicamente de forma heterogénea, com o envolvimento de diversos sistemas de órgãos, podendo originar, em situações mais graves, síndrome da dificuldade respiratória do adulto e disfunção multiorgânica. ${ }^{7}$ Mais frequentemente, os sintomas descritos incluem febre, tosse seca, dispneia, odinofagia, cefaleias, fadiga, diarreia, anosmia e ageusia, ${ }^{8}$ mas casos totalmente assintomáticos têm sido também reportados. ${ }^{9}$ Por outro lado, manifestações dermatológicas relatadas de forma crescente associadas à COVID-19 têm sido alvo de grande interesse, uma vez que o seu reconhecimento adequado poderá ser útil ao estabelecimento diagnóstico e abordagem precoces, ao esclarecimento de mecanismos fisiopatológicos relacionados com a infeção e, nalguns casos, ao fornecimento de orientações prognósticas.

\section{MATERIAL E MÉTODOS}

Foi realizada uma pesquisa na literatura com recurso ao motor de busca PubMed, com o objetivo de identificar artigos indexados à MEDLINE relativos a manifestações dermatológicas associadas à COVID-19. Os termos MeSH inseridos incluíram 'Dermatology', 'COVID-19' e 'SARS-CoV-2', originando um total de 739 resultados. Através da análise dos resumos, foram inicialmente selecionados 267 artigos respeitantes a manifestações cutâneas associadas à infeção por SARS-CoV-2, que por sua vez permitiram identificar outras publicações complementares relevantes. Excluíram-se aqueles relacionados com a utilização de equipamentos de proteção individual, terapêutica farmacológica em contexto da infeção e impacto da infeção no seguimento e abordagem de doentes.

\section{DISCUSSÃO}

\section{Manifestações cutâneas de infeção por SARS-CoV-2}

Os estudos iniciais provenientes da China reportavam frequências muito baixas de manifestações dermatológicas em doentes com COVID-19, pelo que foram parcialmente ignoradas durante vários meses. Em apenas $0,2 \%$ dos 1099 casos confirmados em Wuhan foram reportadas lesões cutâneas. ${ }^{7}$ Com a chegada da pandemia à Europa, muitos médicos de diversas especialidades, incluindo dermatologistas, passaram a constituir equipas que lidavam diretamente com doentes infetados, o que levou a um maior interesse no que concerne a este tipo de sintomas.

A primeira série de casos europeia relativa a manifestações cutâneas da COVID-19, reportada por Recalcati, demonstrou a ocorrência de lesões em 20,4\% ( $n=18)$ dos 88 doentes infetados inseridos no estudo. Os padrões descritos incluíam erupções eritematosas, urticariformes e tipo-varicela. ${ }^{10}$ Pouco depois começaram a ser descritos múltiplos casos clínicos e séries de casos evidenciando lesões semelhantes ${ }^{11-13}$ e outras francamente distintas, como lesões petequiais, tipo-perniose, vasculíticas, livedóides e tipo-eritema polimorfo. ${ }^{14-18}$ Adicionalmente, a emergência de quadros de pitiríase rosea e doença de Kawasaki em doentes infetados sugeriu que o SARS-CoV-2 poderá ter também um papel na reativação de outros vírus, ${ }^{19,20}$ ou na estimulação de mecanismos pro-inflamatórios que conduzem a estas patologias em doentes suscetíveis, como no caso da doença de Kawasaki em que há evidência de produção anómala de interferão do tipo I. ${ }^{21}$

Suchonwanit et al dividiram as manifestações cutâneas da COVID-19 em dois grupos de acordo com prováveis mecanismos fisiopatológicos: exantemas virais, que representam uma resposta imune a moléculas virais; e lesões cutâneas, nomeadamente vasculite e vasculopatia trombótica, secundárias a consequências sistémicas causadas pelo vírus (Tabela 1). ${ }^{22,23}$

Sabe-se que, de um modo geral, as infeções virais conseguem produzir manifestações mais ou menos específicas, tanto pela ação direta em células do hospedeiro, como indiretamente através da resposta do sistema imunitário. ${ }^{24}$ No que respeita às manifestações cutâneas em contexto da infeção por SARS-CoV-2, o recurso à histopatologia, imunohistoquímica e biologia molecular, permitiu inferir mecanismos patogénicos semelhantes aos descritos noutras infeções virais. As lesões cutâneas mais específicas parecem advir do efeito citopático do vírus, já que tanto proteínas virais como recetores utilizados na invasão celular são expressos na pele. ${ }^{25}$ Outras lesões poderão

Tabela 1 - Classificação de manifestações cutâneas associadas à COVID-19 proposta por Suchonwanit et a/21,22

\begin{tabular}{|c|c|c|}
\hline Exantema morbiliforme & \multirow{4}{*}{$\begin{array}{l}\text { Resposta imune de } \\
\text { hipersensibilidade a } \\
\text { moléculas virais. }\end{array}$} & \multirow{4}{*}{$\begin{array}{l}\text { Não fornecem pistas específicas } \\
\text { ao diagnóstico ou prognóstico da } \\
\text { COVID-19. }\end{array}$} \\
\hline Erupção petequial/Máculas purpúricas & & \\
\hline Urticária & & \\
\hline Erupção tipo-varicela & & \\
\hline \multicolumn{3}{|l|}{ Lesões tipo-perniose } \\
\hline Vasculite & \multirow{4}{*}{$\begin{array}{c}\text { Secundário a } \\
\text { consequências sistémicas } \\
\text { causadas diretamente por } \\
\text { COVID-19. }\end{array}$} & \multirow{4}{*}{$\begin{array}{c}\text { Podem ter valor prognóstico, através } \\
\text { da indicação de complicações sérias } \\
\text { da COVID-19 e pela monitorização da } \\
\text { gravidade da doença. }\end{array}$} \\
\hline Livedo reticular/racemoso & & \\
\hline Púrpura retiforme & & \\
\hline Acrocianose e gangrena seca & & \\
\hline
\end{tabular}


representar o resultado de uma resposta imunitária exagerada à infeção, ${ }^{26}$ nomeadamente pela libertação descontrolada de interferão e citocinas pro-inflamatórias também implicadas na progressão da doença e desenvolvimento de quadros mais graves. ${ }^{27}$

Por sua vez, Galván Casas et al, num estudo prospetivo com 375 casos que é, até ao momento, uma das amostras mais representativas, descreveram cinco padrões clínicos principais associados à infeção por SARS-CoV-2 (Tabela 2). ${ }^{28}$ Também Marzano et al realizaram uma revisão de dados publicados na literatura, propondo uma classificação que compreende seis padrões distintos, mas englobáveis nos cinco anteriores (Tabela 2) ${ }^{29}$ os quais passamos a descrever.

\section{Erupções eritematosas e maculopapulares}

As erupções eritematosas e maculopapulares constituem manifestações dermatológicas frequentemente observadas na prática clínica, ocorrendo em contexto de múltiplas condições, nomeadamente reações adversas a fármacos, infeções bacterianas ou virais. Por vezes, a distinção entre uma causa farmacológica ou infeciosa pode ser difícil com base apenas no padrão clínico, sendo que a presença de febre, fadiga e sintomatologia respiratória, complementada por estudo analítico, se associa mais à segunda hipótese. No caso da COVID-19 este tipo de lesões tem sido um dos mais regularmente identificados (Fig. 1A).

$\mathrm{Na}$ primeira série de casos europeia referente a manifestações cutâneas, $77,8 \%(n=14)$ dos doentes demonstravam uma erupção eritematosa, envolvendo maioritariamente o tronco, com pouco prurido associado. ${ }^{10}$ Posteriormente, em diferentes estudos retrospetivos e prospetivos, este tipo de erupção correspondeu à mais frequentemente observada, maioritariamente em doentes sintomáticos. $^{28,30-33}$ Dalal et al descreveram lesões maculopapulares, com envolvimento importante do tronco, poupando as

Tabela 2 - Classificações de manifestações cutâneas associadas à COVID-19 propostas por Galván Casas et a/27 e Marzano et a ${ }^{28}$

\begin{tabular}{cc}
\hline Padrões Clínicos - Galván Casas & Padrões Clínicos - Marzano \\
\hline $\begin{array}{c}\text { Áreas acrais eritemato-edematosas com vesículas ou pústulas } \\
\text { (pseudo-perniose) } \\
\text { Outras erupções vesiculares } \\
\text { Lesões urticariformes }\end{array}$ & Padrão acral tipo-perniose \\
Outras erupções maculopapulares & Exantema papulovesicular \\
Livedo ou necrose & Erupção urticariforme \\
& Erupção eritematosa/maculopapular/morbiliforme \\
& Padrão tipo-livedo reticular/racemoso \\
\hline
\end{tabular}
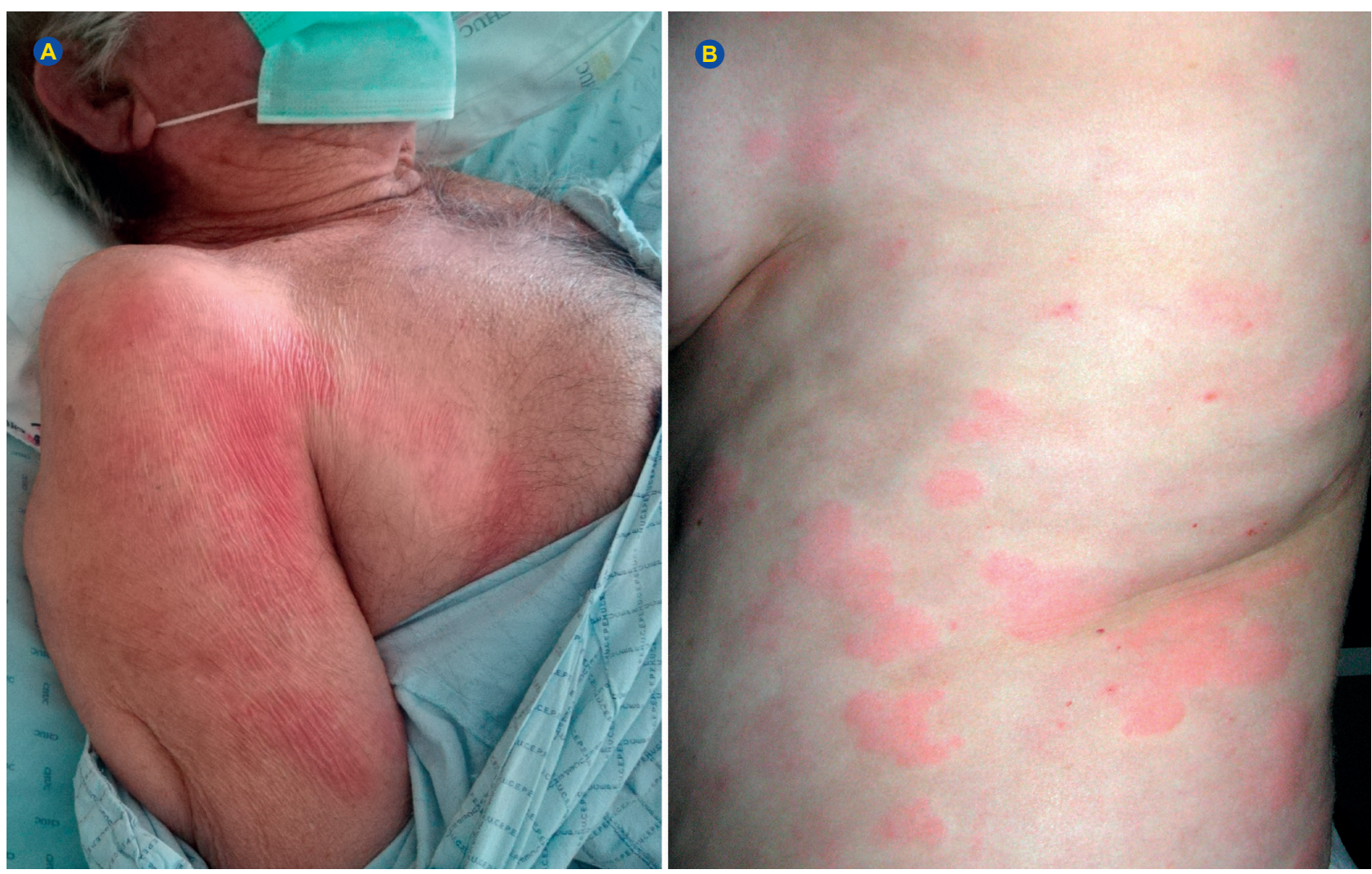

Figura 1 - Diferentes tipos de erupções associadas à COVID-19: (A) Máculas eritematosas com envolvimento do tronco e predomínio flexural; (B) Lesões urticariformes distribuídas maioritariamente pelo tronco 
palmas e plantas, que ocorreram cerca de três dias após o início da febre. ${ }^{32}$ De Giorgi et al observaram uma erupção eritematosa, com distribuição semelhante e uma duração média de três dias, em $70 \%$ dos 53 doentes do estudo. ${ }^{33}$ Galván Casas et al reportaram lesões maculopapulares em 47\% dos 375 doentes: umas com distribuição perifolicular e graus variáveis de descamação, outras mais purpúricas ocupando áreas maiores, e outras localizadas às extremidades mostrando alguma infiltração. De um modo geral a erupção ocorreu concomitantemente ao início de outros sintomas, durou em média nove dias e associou-se a casos de infeção mais grave, que resultaram numa mortalidade de $2 \% .^{28}$

Múltiplos casos semelhantes têm vindo a ser reportados de forma isolada ou em pequenas séries, ${ }^{11,34-38}$ alguns com estudo histopatológico. ${ }^{11,37,38}$ Dois dos três casos reportados por Giannoti et al revelaram achados compatíveis com exantema viral, embora um deles demonstrasse também a presença de microtrombos vasculares e o outro alterações sugestivas de vasculite linfocítica; já o terceiro caso demonstrava uma dermatite de interface com queratinócitos necróticos, sugerindo uma ativação de linfócitos $T$ citotóxicos resultando em dano destas células. ${ }^{37}$ Zengarini et al e Amatore et al descreveram achados histológicos semelhantes a outros exantemas virais, com infiltrado linfocítico perivascular superficial, edema dérmico e espongiose da epiderme. ${ }^{11,38}$

Embora as erupções eritematosas e maculopapulares não constituam uma manifestação específica, quando associadas à COVID-19 parecem surgir em casos mais graves de infeção. ${ }^{28}$ Um dos principais diagnósticos diferenciais corresponde a reações adversas a fármacos, das quais por vezes é impossível distinguir. Tal como acontece, por exemplo, com o vírus Epstein-Barr, devemos ainda considerar a possibilidade destas erupções não surgirem apenas devido ao efeito isolado do vírus, mas também à sensibilização prévia causada por terapêuticas farmacológicas, ${ }^{36}$ nomeadamente antibióticos, habitualmente utlizados neste tipo de doentes.

\section{Erupções urticariformes}

Os quadros de urticária, caracterizados pela presença de placas eritemato-edematosas, pruriginosas e evanescentes (cada lesão tem duração inferior a 24 horas) e/ou angioedema, são frequentemente despoletados por diferentes tipos de infeções, incluindo virais. ${ }^{39}$

A erupção urticariforme foi uma das primeiras manifestações cutâneas a ser descrita em contexto de infeção por SARS-CoV-2, ocorrendo em 16,7\% dos 18 doentes com sintomatologia cutânea observados por Recalcati. As lesões eram ligeiramente pruriginosas, distribuíam-se maioritariamente pelo tronco, e aparentemente não se correlacionavam com a gravidade da infeção. ${ }^{10}$ Posteriormente, lesões semelhantes foram reportadas como manifestação inicial da COVID-19 em quatro doentes. ${ }^{12,40,41}$ Embora dois apresentassem febre, ${ }^{12}$ nenhum demonstrava sintomatologia respiratória aquando da observação inicial, acabando por desenvolvê-la mais tardiamente. A todos foram administrados anti-histamínicos, com resolução da erupção. No mesmo seguimento, Diotallevi et al relataram dois casos com lesões urticariformes concomitantes ao início do quadro respiratório. ${ }^{42}$

Hedou et al, num estudo com 103 doentes, reportaram urticária em apenas dois. ${ }^{30}$ De Giorgi et al observaram-na em $26 \%$ dos 53 doentes de um estudo prospetivo. ${ }^{33}$ No estudo realizado por Gálvan Casas et al, a erupção urticariforme ocorreu em $19 \%$ dos 375 doentes envolvidos. ${ }^{28} \mathrm{Em}$ todos os estudos, as lesões surgiram na fase prodrómica ou em simultâneo aos restantes sintomas, eram pruriginosas, distribuíam-se maioritariamente pelo tronco superior e tinham uma duração média variável entre dois e sete dias. . $^{28,30,33}$

Em conclusão, a erupção urticariforme assemelha-se a quadros de urticária frequentemente observados em contexto infecioso (Fig. 1B). No caso da COVID-19, parece ocorrer na fase inicial, previamente ou em simultâneo à emergência de outros sintomas.

\section{Erupções papulovesiculares}

Diversos agentes infeciosos podem estar implicados no desenvolvimento de lesões vesiculo-bolhosas, localizadas ou generalizadas, com envolvimento mucoso ou não. Mais especificamente, vírus como o herpes simplex ou varicela-zoster conseguem replicar-se nas células epidérmicas dando origem a este tipo de quadros.

Dos primeiros casos de COVID-19 com manifestações dermatológicas descritos, um demonstrava lesões vesiculares semelhantes às da varicela. ${ }^{10}$ Gálvan Casas et al reportaram também a ocorrência de uma erupção vesicular, ligeiramente pruriginosa, em $9 \%$ dos doentes, embora as lesões consistissem maioritariamente em pequenas vesículas monomórficas distribuídas pelo tronco, distintas das geralmente observadas na varicela que se caracterizam por progressão cefalo-caudal e aspeto polimorfo, refletindo diferentes estadios de evolução. Esta erupção ocorreu mais frequentemente em doentes de meia-idade, com quadros moderados de infeção e previamente ao início de sintomas sistémicos, parecendo ser uma manifestação relativamente específica de COVID-19. ${ }^{28}$

Vinte e dois doentes de oito serviços de Dermatologia italianos com exantema papulovesicular tipo-varicela foram relatados por Marzano et al. Dos achados demográficos destacavam-se uma idade média de 60 anos, um tempo de latência médio entre o aparecimento de sintomas sistémicos e o exantema de três dias, uma duração média de oito dias e o facto de todos os doentes já terem tido previamente varicela. Clinicamente as lesões encontravam-se dispersas pelo tegumento, envolvendo sempre o tronco, mas sem atingimento da face ou mucosa. Contrariamente à varicela, não eram umbilicadas e demonstravam pouco prurido, mas, em sete destes doentes, a biópsia cutânea revelou atrofia da epiderme, vacuolização da camada basal com queratinócitos multinucleados e a presença de células disqueratósicas, achados histológicos semelhantes a 
outras infeções virais. ${ }^{13}$

Dois padrões clínicos de erupções vesiculares foram descritos em 29 doentes com COVID-19 num estudo prospetivo: difuso $(75 \%)$, caracterizado por pequenas pápulas, vesículas e pústulas de tamanhos variáveis e estadios diferentes de evolução, distribuídas por mais que uma área corporal, cuja biópsia de dois casos foi semelhante à doença mãos-pés-boca; e localizado (25\%), consistindo em lesões monomórficas, mais pequenas, afetando maioritariamente o tronco. Em nenhum dos quatro doentes testados (três com padrão difuso, um localizado) foi detetado SARS-CoV-2 nas vesículas. ${ }^{43}$

Uma análise histológica mais pormenorizada das lesões de três doentes franceses com erupções vesiculares concluiu que eram semelhantes entre si, mas substancialmente diferentes das observadas na varicela. Embora a pesquisa de SARS-CoV-2 nas vesículas tenha também sido negativa, os achados evocavam um efeito citopático direto do vírus nos queratinócitos, originando acantólise e disqueratose. ${ }^{44}$

\section{Erupções purpúricas e petequiais}

As petéquias, definidas como lesões purpúricas não palpáveis com diâmetro inferior a $4 \mathrm{~mm}$, podem ocorrer no contexto de erupções não vasculíticas inflamatórias ou como um fenómeno secundário durante a evolução de um exantema. ${ }^{29}$

Quadros de erupções petequiais associados à COVID-19 têm vindo a ser descritos. Primeiramente por Joob, com um caso inicialmente diagnosticado como dengue, com trombocitopenia e posterior desenvolvimento de sintomas respiratórios. ${ }^{14}$ Seguidamente, foi reportada a ocorrência de maculas milimétricas, eritemato-purpúricas, por vezes confluentes, localizadas às regiões flexurais numa doente infetada, embora uma eventual relação causal com fármacos não pudesse ser excluída. ${ }^{45}$ Diaz-Guimaraens et al relataram o caso de um doente de 48 anos com sintomatologia respiratória, contagem de plaquetas normal, sem alterações da coagulação, mas d-dímeros aumentados, e lesões semelhantes às de exantema petequial periflexural associado ao parvovírus $\mathrm{B} 19$, que resolveram em cinco dias. A biópsia cutânea revelou infiltrado linfocítico, com extravasamento de eritrócitos e edema papilar focal, mas sem sinais de vasculopatia trombótica. ${ }^{46}$ Também numa doente de 39 anos com COVID-19, sem alterações no hemograma ou coagulação, mas d-dímeros aumentados, foi descrita uma erupção papulo-purpúrica e petequial, simétrica, distribuída pelas nádegas, coxas e axilas, cuja biópsia demonstrou achados similares aos de Diaz-Guimaraens et al, incluindo a ausência de sinais de vasculite. ${ }^{47}$

Este tipo de erupção petequial e/ou purpúrica não palpável, apesar de pouco reportada, parece ocorrer em quadros ligeiros de infeção, contrariamente a lesões purpúricas palpáveis ou livedóides, que habitualmente se associam a situações mais graves e existência de processos vasculopáticos.

\section{Lesões tipo-perniose}

A perniose define-se como uma resposta exagerada da pele ao frio em indivíduos suscetíveis, caracterizando-se por máculas ou pápulas, de coloração eritemato-violácea, localizadas às superfícies acrais, nomeadamente mãos e pés e, pontualmente, nariz e pavilhões auriculares. Engloba um grupo de distúrbios, onde se inclui a sua forma idiopática, mas também a acrocianose, o fenómeno de Raynaud, o livedo reticular, a perniose lúpica, a púrpura retiforme induzida por crioproteínas e a doença por aglutininas frias, podendo associar-se a doença sistémica, nomeadamente auto-imune, genética, neoplásica ou infeciosa. ${ }^{48}$

Os primeiros relatos na Europa de uma possível relação entre perniose/lesões tipo-perniose e COVID-19 provieram de um grupo de mensagens de texto no Whatsapp ${ }^{\circledR}$ constituído por dermatologistas franceses com o objetivo de partilhar informação sobre a pandemia, nomeadamente manifestações dermatológicas, em doentes suspeitos ou infetados por SARS-CoV-2. Através de uma análise, Duong et al identificaram 146 imagens de lesões tipo-perniose, concluindo que, tal como acontecia com os casos de anosmia, este fosse um sintoma maioritariamente reportado por doentes tratados em ambulatório, ou seja, com quadros ligeiros de infeção. ${ }^{15}$ Similarmente, Piccolo et al, num estudo com 63 casos, descreveram que estes doentes eram geralmente indivíduos jovens, saudáveis, com sintomas sistémicos ocasionais e ligeiros precedendo as manifestações dermatológicas. Clinicamente as lesões eram dolorosas e/ou pruriginosas, afetavam maioritariamente os dedos e plantas dos pés, dividindo-se em dois padrões: eritemato-edematosas e bolhosas, tendo assim uma apresentação muito semelhante à perniose, exceto pela ausência da exposição ao frio. ${ }^{49}$

Seguidamente um número crescente de quadros semeIhantes começou a ser reportado em diferentes países, reforçando a associação com a COVID-19.42,50-59 De um modo geral, as lesões ocorriam em jovens previamente saudáveis, podendo estar associadas a sintomatologia sistémica ou não. Caso estivessem, esta era habitualmente ligeira e as manifestações cutâneas ocorriam posteriormente. Caracterizavam-se por pápulas e máculas eritemato-violáceas associadas, por vezes, a lesões bolhosas e edema dos dedos, sendo os pés mais frequentemente afetados que as mãos. Mais raramente, as lesões apresentavam uma conformação em alvo, assemelhando-se às lesões do eritema polimorfo, condição despoletada por infeções virais como o herpes simplex. Tal fato reforçou a hipótese do próprio SARS-CoV-2 ser também um desencadeante possível desta dermatose, na sua forma minor (sem componente mucoso). ${ }^{18,60}$

Um pormenor importante, comum a praticamente todos os estudos e relatos, foi o reduzido número casos de infeção confirmados, quer porque não havia informação ou o teste não foi realizado, quer porque foi negativo. Para a última situação, possíveis explicações foram propostas: os achados dermatológicos não estarem relacionados com a COVID-19; tratarem-se de falsos-negativos; ou o teste ter 
sido realizado após clearance do vírus, já que as lesões tipo-perniose ocorreram numa fase mais tardia da infeção. ${ }^{53}$ Curiosamente, com a introdução de testes serológicos, foram reportados dois casos com lesões deste tipo e zaragatoa nasofaríngea negativa, que demonstraram IgG específica positiva para SARS-CoV-2, reforçando a última hipótese. ${ }^{61,62}$

Em termos histológicos, as lesões tipo-perniose caracterizavam-se, de uma forma geral, por infiltrado linfocítico dérmico periecrino e perivascular, sendo que os vasos envolvidos apresentavam ativação de células endoteliais e extravasamento de eritrócitos, mas raramente com dano vascular franco. ${ }^{63-66}$ Achados idênticos são observados em lesões de perniose lúpica, o que levou à suspeita de semeIhança entre os mecanismos fisiopatológicos subjacentes. A associação entre a infeção e este tipo de lesões foi corroborada por um estudo recente, em que sete casos com biópsia cutânea lesional demonstravam positividade para a proteína spike do SARS-CoV-2 no estudo imunohistoquími$\mathrm{co.}^{66}$

Assim, colocou-se a hipótese de doentes mais jovens demonstrarem uma reação imune exuberante mediada por interferão do tipo I, conseguindo conter a infeção viral de forma prematura, permitindo um curso mais favorável. Esta mesma resposta parece ser a responsável pela ocorrência deste tipo de manifestações dermatológicas..$^{59,64,65}$

Reforça-se que as lesões tipo-perniose não devem ser confundidas com as de acro-isquemia, causadas por trombose de pequenos vasos da pele, e que são habitualmente observadas em doentes com quadros graves de COVID-19 (Fig. 2A).

\section{Lesões livedóides e acro-isquemia}

Lesões livedóides são resultantes de microvasculopatia trombótica oclusiva, apresentando diferentes níveis de gravidade. ${ }^{29}$ Podem associar-se a doença sistémica ou não, sendo que no primeiro caso se incluem condições hematológicas, reumáticas, cardiovasculares e infeciosas. ${ }^{67} \mathrm{Em}$ contexto de infeção a ocorrência de trombose deve-se à invasão das células endoteliais pelo agente infecioso ou à ativação da cascata da coagulação, que pode variar entre fenómenos subclínicos, apenas com aumento ligeiro dos marcadores associados à formação de fibrina e trombina, e coagulação intravascular disseminada (CID). ${ }^{68}$

Manalo et al reportaram livedo reticular assintomático, transitório, em dois doentes com quadros ligeiros de COVID-19, pressupondo uma correlação entre o grau de oclusão provocada por microtromboses e a gravidade da infeção, embora com algumas limitações, já que não tiveram acesso a estudo analítico ou biópsia cutânea. ${ }^{17}$

Galván Casas et al descreveram a ocorrência de diferentes graus de lesões livedóides e necróticas em $6 \%$ dos casos, sugerindo doença vascular oclusiva. De um modo geral, correspondiam a pessoas mais idosas, com doença mais severa e uma mortalidade associada de $10 \%$, embora também existissem casos de livedo reticular transitório e sem necessidade de hospitalização. ${ }^{28}$

Lesões de acro-isquemia, caracterizadas por cianose, bolhas hemorrágicas e gangrena seca dos dedos dos pés e das mãos resultantes de um quadro de hipercoaguabilidade e CID, foram inicialmente reportadas em doentes graves com COVID-19, permitindo estabelecer uma relação preliminar entre a infeção e a ocorrência deste fenómeno. Achados provenientes de autópsias de cinco doentes críticos infetados, dos quais três tinham lesões cutâneas livedóides ou acro-isquémicas, documentaram a presença de dano microvascular trombótico associado à deposição de proteínas do complemento, co-localizadas com glicoproteínas específicas do SARS-CoV-2. Tal veio reforçar a teoria de um potencial papel do vírus na ativação do complemento e interação com as vias de coagulação, sugerindo a investigação de biomarcadores associados a dano microvascular

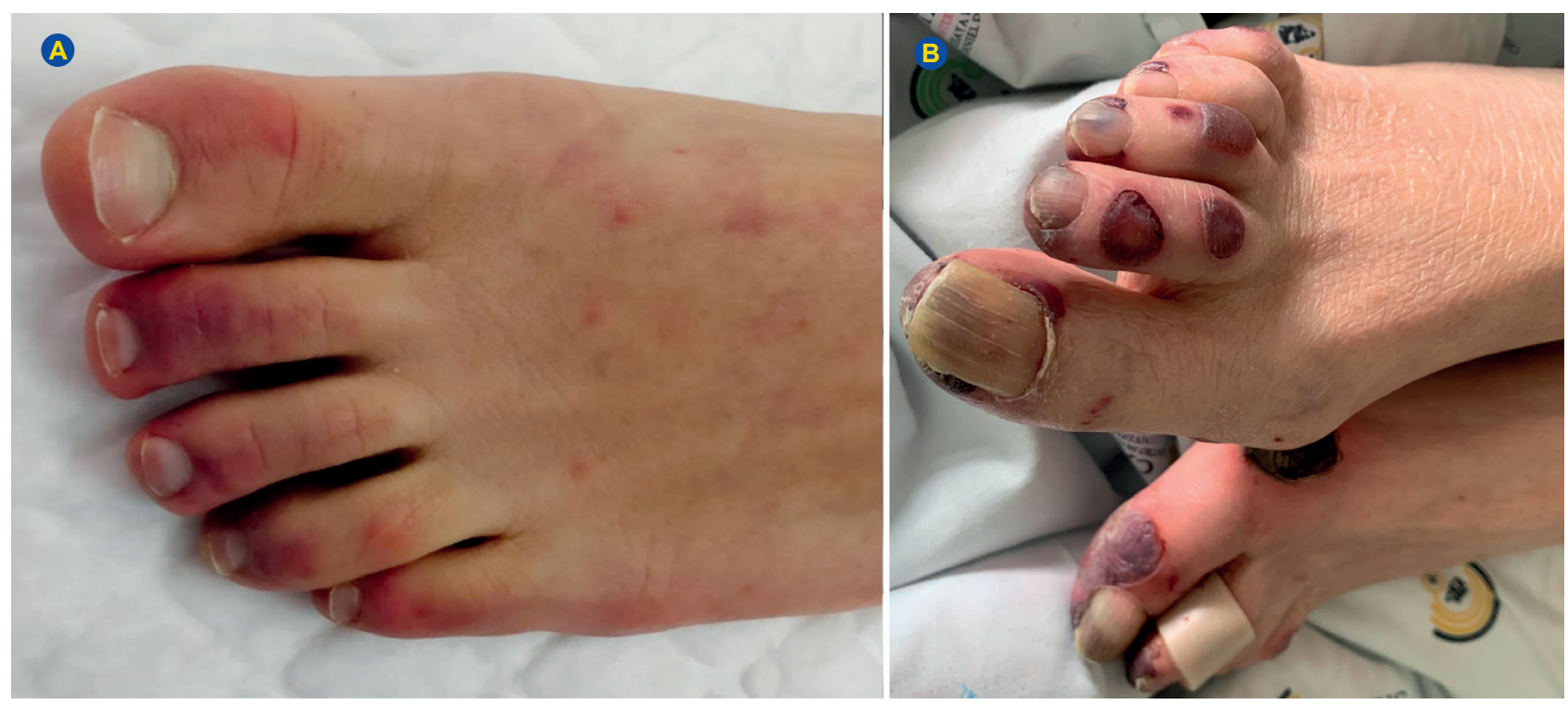

Figura 2 - Diferentes tipos de lesões acrais associadas à COVID-19: (A) Máculas eritemato-violáceas de localização distal nos dedos dos pés, compatíveis com lesões tipo-perniose; (B) Bolhas hemorrágicas e necrose seca dos dedos dos pés, compatíveis com acro-isquemia 
e trombose, como d-dímeros, fibrinogénio, fator VIII, anticorpos anti-fosfolipídicos, proteína $\mathrm{C}$ reativa, proteínas do complemento e citocinas pro-inflamatórias. ${ }^{69}$ Outros casos de acro-isquemia foram relatados (Fig. 2B), ${ }^{16,59,70-73}$ sendo que nove tinham informação respeitante a achados analíticos: todos demonstravam elevação dos d-dímeros e da proteína C-reativa, ${ }^{59,70-73}$ um apresentava trombocitopenia ${ }^{71}$ e sete aumento do fibrinogénio. ${ }^{59,72}$ De um modo geral, as biópsias cutâneas revelaram uma vasculopatia trombótica pauci-inflamatória, embora não tenha sido detetado RNA viral. ${ }^{59,69}$ Este tipo de manifestações dermatológicas parece advir de uma desregulação do sistema imunitário, com respostas de interferão do tipo I diminuídas e ativação do complemento, originando lesão microvascular e estados pro-coagulantes, associando-se a prognósticos desfavoráveis. ${ }^{59}$

\section{CONCLUSÃO}

Em relativamente pouco tempo, as manifestações dermatológicas associadas à COVID-19 deixaram de ser praticamente inexistentes para passarem a constituir possíveis auxiliares ao diagnóstico precoce e, em situações mais limitadas, estratificação de risco. Mais especificamente, o reconhecimento de padrões clínicos, como os descritos, pode ser útil na suspeição de infeção por SARS-CoV-2 nomeadamente em indivíduos oligossintomáticos ou nos quais não é possível a realização imediata de testes confirmatórios.

De referir que, apesar de tudo, nenhum dos quadros de erupções relatados (eritematosa/maculopapular, urticariforme, papulovesicular, purpúrica não palpável/petequial) é exclusivo à COVID-19, podendo ser encontrados noutras infeções virais. Apesar disso, a infeção por SARS-CoV-2 deve ser atualmente considerada na prática clínica como uma possível etiologia perante erupções deste tipo. Por outro lado, acabam por ser raras em quadros respiratórios como o da gripe, um importante diagnóstico diferencial da COVID-19. Já no que diz respeito a prognóstico, as opi-

\section{REFERÊNCIAS}

1. World Health Organization. WHO characterizes COVID-19 as a pandemic - 11 March 2020. [Consultado 2020 jul 13]. Disponível em: https://www.who.int/emergencies/diseases/novel-coronavirus-2019/ events-as-they-happen.

2. Johns Hopkins Coronavirus Resource Center. COVID-19 Data in Motion. [Consultado 2020 nov 20]. Disponível em: https://coronavirus. jhu.edu.

3. Direção-Geral da Saúde. COVID-19. [consultado 2020 nov 20]. Disponível em: http://covid-19.min-saude.pt.

4. Delfino M, Guida M, Patri A, Spirito L, Gallo L, Fabbrocini G. SARSCoV-2 possible contamination of genital area: implications for sexual and vertical transmission routes. J Eur Acad Dermatol Venereol. 2020;34:e364-5.

5. Lauer SA, Grantz KH, Bi Q, Jones FK, Zheng Q, Meredith HR, et al. The incubation period of coronavirus disease 2019 (COVID-19) from publicly reported confirmed cases: estimation and application. Ann Intern Med. 2020;172:577-82

6. Wang $Y Y$, Jin $Y H$, Ren XQ, Li YR, Zhang XC, Zeng $X T$, et al. Updating the diagnostic criteria of COVID-19 "suspected case" and "confirmed case" is necessary. Mil Med Res. 2020;7:17.

7. Guan WJ, Ni ZY, Hu Y, Liang WH, Ou CQ, He JX, et al. Clinical characteristics of coronavirus disease 2019 in China. N Engl J Med. niões divergem: alguns estudos indicam que a erupção maculopapular se relaciona com cursos de doença mais grave, mas a maioria refere que, tal como nas restantes erupções, não há qualquer correlação com a gravidade da infeção. Quanto às lesões tipo-perniose, não só podem ser úteis ao diagnóstico de indivíduos assintomáticos, como parecem indicar prognóstico favorável. Em contraste, as lesões livedóides e de acro-isquemia ocorrem em doentes mais idosos, associando-se a quadros de hipercoaguabilidade e a prognósticos mais sombrios.

Tal como na maioria dos temas inerentes a esta pandemia, ainda muito está por descobrir no que respeita a manifestações dermatológicas, havendo necessidade de mais estudos. Novos dados informativos são constantemente relatados, sendo de importância primordial o acompanhamento dessas atualizações por parte da comunidade médica e científica.

\section{PROTEÇÃO DE PESSOAS E ANIMAIS}

Os autores declaram que os procedimentos seguidos estavam de acordo com os regulamentos estabelecidos pelos responsáveis da Comissão de Investigação Clínica e Ética e de acordo com a Declaração de Helsínquia da Associação Médica Mundial atualizada em 2013.

\section{CONFIDENCIALIDADE DOS DADOS}

Os autores declaram ter seguido os protocolos do seu centro de trabalho acerca da publicação de dados.

\section{CONFLITOS DE INTERESSE}

Os autores declaram não ter conflitos de interesses relacionados com o presente trabalho.

\section{FONTES DE FINANCIAMENTO}

Este trabalho não recebeu qualquer tipo de suporte financeiro de nenhuma entidade no domínio público ou privado.

2020;382:1708-20.

8. Wang D, Hu B, Hu C, Zhu F, Liu X, Zhang J, et al. Clinical characteristics of 138 hospitalized patients with 2019 novel coronavirus-infected pneumonia in Wuhan, China. JAMA. 2020;323:1061-9.

9. Bai Y, Yao L, Wei T, Tian F, Jin DY, Chen L, et al. Presumed asymptomatic carrier transmission of COVID-19. JAMA. 2020;323:1406-7.

10. Recalcati S. Cutaneous manifestations in COVID-19: a first perspective. J Eur Acad Dermatol Venereol. 2020;34:e212-3.

11. Zengarini C, Orioni G, Cascavilla A, Horna Solera C, Fulgaro C, Misciali C, et al. Histological pattern in COVID-19-induced viral rash. J Eur Acad Dermatol Venereol. 2020 (in press). doi:10.1111/jdv.16569.

12. van Damme $C$, Berlingin E, Saussez S, Accaputo O. Acute urticaria with pyrexia as the first manifestations of a COVID-19 infection. J Eur Acad Dermatol Venereol. 2020;34:e300-1.

13. Marzano AV, Genovese G, Fabbrocini G, Pigatto P, Monfrecola G, Piraccini BM, et al. Varicella-like exanthem as a specific COVID-19associated skin manifestation: multicenter case series of 22 patients. J Am Acad Dermatol. 2020;83:280-5.

14. Joob B, Wiwanitkit V. COVID-19 can present with a rash and be mistaken for dengue. J Am Acad Dermatol. 2020;82:e177.

15. Duong TA, Velter C, Rybojad M, Comte C, Bagot M, Sulimovic L, et al. Did Whatsapp $((R))$ reveal a new cutaneous COVID-19 manifestation? J 
Eur Acad Dermatol Venereol. 2020;34:e348-50.

16. Mazzotta F, Troccoli T, Bonifazi E. A new vasculitis at the time of COVID-19. Eur J Pediat Dermatol. 2020:30:75-8.

17. Manalo IF, Smith MK, Cheeley J, Jacobs R. A dermatologic manifestation of COVID-19: transient livedo reticularis. J Am Acad Dermatol. 2020;83:700.

18. Janah $\mathrm{H}$, Zinebi A, Elbenaye J. Atypical erythema multiforme palmar plaques lesions due to Sars-Cov-2. J Eur Acad Dermatol Venereol. 2020;34:e373-5.

19. Ehsani $A H$, Nasimi M, Bigdelo Z. Pityriasis rosea as a cutaneous manifestation of COVID-19 infection. J Eur Acad Dermatol Venereol. 2020; 34:e436-7.

20. Dursun R, Temiz SA. The clinics of HHV-6 infection in COVID-19 pandemic: pityriasis rosea and Kawasaki disease. Dermatol Ther. 2020:e13730.

21. Berthelot JM, Drouet L, Liote F. Kawasaki-like diseases and thrombotic coagulopathy in COVID-19: delayed over-activation of the STING pathway? Emerg Microbes Infect. 2020;9:1514-22.

22. Suchonwanit $P$, Leerunyakul K, Kositkuljorn C. Cutaneous manifestations in COVID-19: lessons learned from current evidence. J Am Acad Dermatol. 2020;83:e57-e60.

23. Suchonwanit $\mathrm{P}$, Leerunyakul K, Kositkuljorn C. Diagnostic and prognostic values of cutaneous manifestations in COVID-19. Dermatol Ther. 2020:e13650.

24. Criado PR, Abdalla BM, de Assis IC, Mello C, Caputo GC, Vieira IC. Are the cutaneous manifestations during or due to SARS-CoV-2 infection/ COVID-19 frequent or not? Revision of possible pathophysiologic mechanisms. Inflamm Res. 2020;69:745-56.

25. Xiaotong $X$, Zihao M, Zhenzhen W, Zheng P, Hong L, Furen Z. High expression of ACE2 on the keratinocytes reveals skin as a potential target for SARS-CoV-2. J Invest Dermatol. 2020 (in press). doi: 10.1016/j.jid.2020.05.087.

26. Kanitakis J, Lesort C, Danset M, Jullien D. Chilblain-Like acral lesions during the COVID-19 pandemic ("COVID toes"): histologic, immunofluorescence and immunohistochemical study of 17 cases. J Am Acad Dermatol. 2020;83:870-5.

27. Mehta P, McAuley DF, Brown M, Sanchez E, Tattersall RS, Manson $\mathrm{JJ}$, et al. COVID-19: consider cytokine storm syndromes and immunosuppression. Lancet. 2020;395:1033-4.

28. Galvan Casas C, Catala A, Carretero Hernandez G, Rodriguez-Jimenez P, Fernandez-Nieto D, Rodriguez-Villa Lario A, et al. Classification of the cutaneous manifestations of COVID-19: a rapid prospective nationwide consensus study in Spain with 375 cases. Br J Dermatol. 2020;183:717.

29. Marzano AV, Cassano N, Genovese G, Moltrasio C, Vena GA. Cutaneous manifestations in patients with COVID-19: A preliminary review of an emerging issue. Br J Dermatol. 2020;183:431-42.

30. Hedou M, Carsuzaa F, Chary E, Hainaut E, Cazenave-Roblot F, Masson Regnault M. Comment on "Cutaneous manifestations in COVID-19: a first perspective" by Recalcati S. J Eur Acad Dermatol Venereol. 2020;34:e299-e300.

31. Rubio-Muniz CA, Puerta-Pena M, Falkenhain-Lopez D, ArroyoAndres J, Agud-Dios M, Rodriguez-Peralto JL, et al. The broad spectrum of dermatological manifestations in COVID-19. Clinical and histopathological features learned from a series of 34 cases. J Eur Acad Dermatol Venereol. 2020;34:e574-6.

32. Dalal A, Jakhar D, Agarwal V, Beniwal R, Jakhar D, Agarwal V, et al. Dermatological findings in SARS-CoV-2 positive patients: an observational study from North India. Dermatol Ther. 2020:e13849.

33. De Giorgi V, Recalcati S, Jia Z, Chong W, Ding R, Deng Y, et al. Cutaneous manifestations related to coronavirus disease 2019 (COVID-19): a prospective study from China and Italy. J Am Acad Dermatol. 2020;83:674-5.

34. Avellana Moreno R, Estela Villa LM, Avellana Moreno V, Estela Villa C, Moreno Aparicio MA, Avellana Fontanella JA. Cutaneous manifestation of COVID-19 in images: a case report. J Eur Acad Dermatol Venereol. 2020;34:e307-9.

35. Mahe A, Birckel E, Krieger S, Merklen C, Bottlaender L. A distinctive skin rash associated with coronavirus disease 2019? J Eur Acad Dermatol Venereol. 2020;34:e246-7.

36. Najarian DJ. Morbilliform exanthem associated with COVID-19. JAAD Case Rep. 2020;6:493-4.

37. Gianotti R, Veraldi S, Recalcati S, Cusini M, Ghislanzoni M, Boggio F, et al. Cutaneous clinico-pathological findings in three COVID-19-positive patients observed in the metropolitan area of Milan, Italy. Acta Derm
Venereol. 2020;100:adv00124.

38. Amatore F, Macagno N, Mailhe M, Demarez B, Gaudy-Marqueste C, Grob JJ, et al. SARS-CoV-2 infection presenting as a febrile rash. J Eur Acad Dermatol Venereol. 2020;34:e304-6.

39. Zuberbier T, Aberer W, Asero R, Abdul Latiff AH, Baker D, BallmerWeber $B$, et al. The EAACI/GA(2)LEN/EDF/WAO guideline for the definition, classification, diagnosis and management of urticaria. Allergy. 2018;73:1393-414

40. Henry D, Ackerman M, Sancelme E, Finon A, Esteve E. Urticarial eruption in COVID-19 infection. J Eur Acad Dermatol Venereol. 2020;34:e244-5.

41. Hassan K. Urticaria and angioedema as a prodromal cutaneous manifestation of SARS-CoV-2 (COVID-19) infection. BMJ Case Rep. 2020;13:e236981.

42. Diotallevi F, Campanati A, Bianchelli T, Bobyr I, Luchetti MM, Marconi $B$, et al. Skin involvement in SARS-CoV-2 infection: case series. J Med Virol. 2020;92:2332-4.

43. Fernandez-Nieto D, Ortega-Quijano D, Jimenez-Cauhe J, Burgos-Blasco $P$, de Perosanz-Lobo D, Suarez-Valle A, et al. Clinical and histological characterization of vesicular COVID-19 rashes: a prospective study in a tertiary care hospital. Clin Exp Dermatol. 2020 (in press). doi: 10.1111/ ced.14277.

44. Mahe A, Birckel E, Merklen C, Lefebvre P, Hannedouche C, Jost M, et al. Histology of skin lesions establishes that the vesicular rash associated with COVID-19 is not "varicella-like". J Eur Acad Dermatol Venereol. 2020;34:e559-61.

45. Jimenez-Cauhe J, Ortega-Quijano D, Prieto-Barrios M, Moreno-Arrones OM, Fernandez-Nieto D. Reply to "COVID-19 can present with a rash and be mistaken for dengue": Petechial rash in a patient with COVID-19 infection. J Am Acad Dermatol. 2020;83:e141-2.

46. Diaz-Guimaraens B, Dominguez-Santas M, Suarez-Valle A, PindadoOrtega C, Selda-Enriquez G, Bea-Ardebol S, et al. Petechial skin rash associated with severe acute respiratory syndrome coronavirus 2 infection. JAMA Dermatol. 2020;156:820-2.

47. Larrondo J, Cabrera R, Gosch M, Larrondo F, Aylwin M, Castro A. Papular-purpuric exanthem in a COVID-19 patient: clinical and dermoscopic description. J Eur Acad Dermatol Venereol. 2020;34:e5702.

48. Crowson AN, Magro CM. Idiopathic perniosis and its mimics: a clinical and histological study of 38 cases. Hum Pathol. 1997;28:478-84.

49. Piccolo V, Bassi A. Acral findings during the COVID-19 outbreak: chilblain-like lesions should be preferred to acro-ischemic lesions. J Am Acad Dermatol. 2020;83:e231.

50. Bouaziz JD, Duong T, Jachiet M, Velter C, Lestang P, Cassius C, et al. Vascular skin symptoms in COVID-19: a french observational study. J Eur Acad Dermatol Venereol. 2020; 34:e451-2.

51. Colonna C, Genovese G, Monzani NA, Picca M, Boggio F, Gianotti R, et al. Outbreak of chilblain-like acral lesions in children in the metropolitan area of Milan, Italy, during the COVID-19 pandemic. J Am Acad Dermatol. 2020;83:965-9.

52. Estebanez A, Perez-Santiago L, Silva E, Guillen-Climent S, GarciaVazquez A, Ramon MD. Cutaneous manifestations in COVID-19: a new contribution. J Eur Acad Dermatol Venereol. 2020;34:e250-1.

53. Freeman EE, McMahon DE, Lipoff JB, Rosenbach M, Kovarik C, Takeshita J, et al. Pernio-like skin lesions associated with COVID-19: a case series of 318 patients from 8 countries. J Am Acad Dermatol. 2020;83:486-92

54. Guarneri C, Rullo EV, Pavone P, Berretta M, Ceccarelli M, Natale A, et al. Silent COVID-19: what your skin can reveal. Lancet Infect Dis. 2020 (in press). doi: 10.1016/S1473-3099(20)30402-3.

55. Mastrolonardo M, Romita P, Bonifazi E, Giuffrida R, Lotti T, Foti C, et al. The management of the outbreak of acral skin manifestations in asymptomatic children during COVID-19 era. Dermatol Ther 2020:e13617.

56. Recalcati S, Barbagallo T, Frasin LA, Prestinari F, Cogliardi A, Provero MC, et al. Acral cutaneous lesions in the time of COVID-19. J Eur Acad Dermatol Venereol. 2020;34:e346-7.

57. Saenz Aguirre A, De la Torre Gomar FJ, Roses-Gibert P, Gimeno Castillo J, Martinez de Lagran Alvarez de Arcaya Z, Gonzalez-Perez R. Novel outbreak of acral lesions in times of COVID-19: A description of 74 cases from a tertiary university hospital in Spain. Clin Exp Dermatol. 2020;45:1065-7

58. Tosti G, Barisani A, Queirolo P, Pennacchioli E, Villa L, Lodeserto AM, et al. Skin signs resembling vascular acrosyndromes during the COVID-19 outbreak in Italy. Clin Exp Dermatol. 2020;45:757-8.

59. Magro C, Mulvey JJ, Laurence J, Sanders S, Crowson N, Grossman M, 
et al. The differing pathophysiologies that underlie COVID-19 associated perniosis and thrombotic retiform purpura: a case series. $\mathrm{Br} \mathrm{J}$ Dermatol. 2020 (in press). doi: 10.1111/bjd.19415.

60. Torrelo A, Andina D, Santonja C, Noguera-Morel L, Bascuas-Arribas M, Gaitero-Tristan J, et al. Erythema multiforme-like lesions in children and COVID-19. Pediatr Dermatol. 2020;37:442-6.

61. Kerber AA, Soma DB, Youssef MJ. Chilblains-like dermatologic manifestation of COVID-19 diagnosed by serology via multidisciplinary virtual care. Int J Dermatol. 2020;59:1024-5.

62. Colonna C, Spinelli F, Monzani NA, Ceriotti F, Gelmetti C. Chilblains in children in the time of Covid-19: new evidence with serology assay. Pediatr Dermatol. 2020;37:1000-1.

63. Locatelli AG, Robustelli Test E, Vezzoli P, Carugno A, Moggio E, Consonni $L$, et al. Histologic features of long-lasting chilblain-like lesions in a paediatric COVID-19 patient. J Eur Acad Dermatol Venereol. 2020;34:e365-8.

64. Rodriguez-Villa Lario A, Vega-Diez D, Gonzalez-Canete M, GomezZubiaur A, Perez-Mesonero R, Bandini M, et al. Histological findings in chilblain-lupus like COVID lesions: in search of an answer to understand their etiology. J Eur Acad Dermatol Venereol. 2020;34:e572-4.

65. Kolivras A, Dehavay F, Delplace D, Feoli F, Meiers I, Milone L, et al. Coronavirus (COVID-19) infection-induced chilblains: a case report with histopathologic findings. JAAD Case Rep. 2020;6:489-92.

66. Colmenero I, Santonja C, Alonso-Riaño M, Noguera-Morel L, Hernández-Martín A, Andina D, et al. SARS-CoV-2 endothelial infection causes COVID-19 chilblains: histopathological, immunohistochemical and ultrastructural study of seven paediatric cases. $\mathrm{Br} J$ Dermatol.
2020;183:729-37

67. Elmas OF, Demirbas A, Ozyurt K, Atasoy M, Tursen U. Cutaneous manifestations of COVID-19: a review of the published literature. Dermatol Ther. 2020:e13696.

68. van Gorp EC, Suharti C, ten Cate H, Dolmans WM, van der Meer JW, ten Cate JW, et al. Review: infectious diseases and coagulation disorders. J Infect Dis. 1999;180:176-86.

69. Magro C, Mulvey JJ, Berlin D, Nuovo G, Salvatore S, Harp J, et al. Complement associated microvascular injury and thrombosis in the pathogenesis of severe COVID-19 infection: a report of five cases. TransI Res. 2020;220:1-13.

70. Balestri R, Termine S, Rech G, Girardelli CR. Late onset of acra necrosis after SARS-CoV-2 infection resolution. J Eur Acad Dermatol Venereol. 2020; 34:e448-9..

71. Calvao J, Relvas M, Pinho A, Brinca A, Cardoso JC. Acro-ischemia and COVID-19 infection: clinical and histopathological features. J Eur Acad Dermatol Venereol. 2020 (in press). doi:10.1111/jdv.16667.

72. Del Giudice P, Boudoumi D, Le Guen B, Reverte M, Gutnecht J, Lacour JP, et al. Catastrophic acute bilateral lower limbs necrosis associated with COVID-19 as a likely consequence of both vasculitis and coagulopathy. J Eur Acad Dermatol Venereol. 2020 (in press). doi:10.1111/jdv.16763.

73. Bitar C, Chan MP, Harms PW, Fullen DR, Gudjonsson JE, Eshaq M, et al. Cutaneous manifestations of hospitalized COVID-19 patients: A report of six cases with clinicopathologic features and viral RNA in situ hybridization. J Eur Acad Dermatol Venereol. 2020 (in press). doi: $10.1111 / j \mathrm{dv} .16741$. 